\title{
A Unified Bilevel Programming Framework for Price-Based Market Clearing Under Marginal Pricing
}

\author{
Ricardo Fernández-Blanco, Student Member, IEEE, José M. Arroyo, Senior Member, IEEE, and \\ Natalia Alguacil, Senior Member, IEEE
}

\begin{abstract}
Market clearing in restructured power systems is mostly implemented through an offer-based setting with the goal of maximizing social welfare. This approach leads to sound results from an economic viewpoint when generation offers reflect true production costs. However, offers may significantly differ from actual costs in practice, thus yielding undesired distortion. Under a marginal pricing scheme, this paper presents a general bilevel programming framework for alternative market-clearing procedures dependent on market-clearing prices rather than on offers. The resulting nonlinear mixed-integer bilevel programming formulation is transformed into an equivalent single-level mixed-integer linear program suitable for efficient off-the-shelf software. The bilevel formulation is investigated through a particular instance of price-based market clearing driven by consumer payment minimization. This problem has recently received considerable attention due to the open challenges posed from both modeling and computational perspectives. Numerical results are provided to illustrate the performance of the proposed approach.
\end{abstract}

Index Terms-Bilevel programming, consumer payment minimization, marginal pricing, offer cost minimization, price-based market-clearing procedure.

\section{NOMENCLATURE}

$\begin{array}{ll}\text { A. Indices } & \\ b & \text { Offer block index. } \\ j & \text { Generating unit index. } \\ t & \text { Time period index. }\end{array}$

\section{B. Sets}

$J$

$T$

$X$

$\Pi_{j t}$

$\Omega_{j t}$

Index set of generating units.

Index set of time periods.

Feasibility set for scheduling variables $x$.

Feasibility set for the dispatching variables associated with unit $j$ in period $t$.

Feasibility set for the scheduling variables associated with unit $j$ in period $t$.

Manuscript received February 23, 2011; revised May 20, 2011; accepted June 24, 2011. Date of publication August 12, 2011; date of current version January 20, 2012. This work was supported in part by the Ministry of Science of Spain, under CICYT Project ENE2009-07836 and in part by the Junta de Comunidades de Castilla-La Mancha, under Project POII11-0130-2055. Paper no. TPWRS00164-2011.

The authors are with the Departamento de Ingeniería Eléctrica, Electrónica, Automática y Comunicaciones, E.T.S.I. Industriales, Universidad de Castilla-La Mancha, Ciudad Real E-13071, Spain (e-mail: Ricardo.FCarramolino@uclm.es; JoseManuel.Arroyo@uclm.es; Natalia.Alguacil@uclm.es).

Digital Object Identifier 10.1109/TPWRS.2011.2161348

\section{Functions}

$F(\cdot) \quad$ Objective function of the market-clearing procedure.

$f(\cdot) \quad$ Objective function of the economic dispatch.

$G^{e}(\cdot) \quad$ Constrained functions associated with power balance equations.

$G^{i}(\cdot) \quad$ Constrained functions associated with the inequalities representing the system operation.

$H(\cdot) \quad$ Constrained functions associated with revenues from selling energy.

$L(\cdot) \quad$ Lagrangian function of the lower-level problem.

$\Gamma(\cdot) \quad$ Functions associated with the definition of market-clearing prices.

D. Constants

$D_{t}$

Demand in period $t$.

$D T_{j}$

$O_{b j t}^{E}$

$O_{j t}^{N L}$

$O_{j t}^{S U}$

$\bar{P}_{j t}$

$\underline{P}_{j t}$

$R D_{j}$

$R U_{j}$

$S_{j}^{0}$

$S D_{j}$

$S U_{j}$

$U T_{j}$

E. Variables

$o_{j t}^{E}$

Minimum down time of unit $j$.

Price of the $b$ th energy block offered by unit $j$ in period $t$.

No-load offer price of unit $j$ in period $t$.

Start-up offer price of unit $j$ in period $t$.

Upper bound for the power output of unit $j$ in period $t$.

Lower bound for the power output of unit $j$ in period $t$.

Ramp-down limit of unit $j$.

Ramp-up limit of unit $j$.

Number of periods unit $j$ has been offline prior to the first period of the time span (end of period 0).

Shut-down ramp limit of unit $j$.

Start-up ramp limit of unit $j$.

Minimum up time of unit $j$.

$p_{j t} \quad$ Power output of unit $j$ in period $t$.

$s_{j t} \quad$ Payment for the start-up of unit $j$ in period $t$.

$v_{j t} \quad$ Binary variable that is equal to 1 if unit $j$ is scheduled on in period $t$, being 0 otherwise.

$\lambda_{t} \quad$ Market-clearing price in period $t$. 


\section{F. Vectors \\ $x \quad$ Scheduling variables. \\ $y \quad$ Dispatching variables. \\ $\lambda \quad$ Market-clearing prices. \\ $\mu \quad$ Lagrange multipliers associated with the inequality constraints of the economic dispatch. \\ $\mu_{j t}$ \\ Lagrange multipliers associated with the constraints on the dispatching variables of unit $j$ in period $t$.}

\section{INTRODUCTION}

$\mathbf{I}$ $\mathrm{N}$ the framework of the nowadays competitive power industry, this paper considers a pool-based electricity market for energy. The independent system operator (ISO) receives energy offers from producers and energy bids from consumers, and determines, for every hour, the market-clearing price, the power productions, and the consumption levels.

The market-clearing procedure used by the ISO is typically formulated as a unit commitment problem [1]-[3] in which generation offers replace production costs and consumption bids are taken into account. In this problem, the objective function to be maximized is referred to as the declared social welfare. When the demand is inelastic, the objective function only includes generation offer information and the resulting problem becomes an offer cost minimization. Market-clearing prices resulting from the unit commitment problem are used for market settlement.

Under the assumption of perfect competition, the optimal market-clearing solution is equal to the equilibrium solution where the marginal value to consumers is equal to the marginal cost to producers. This equilibrium maximizes the social welfare, which is the sum of the consumer surplus and the producer surplus. As a consequence, suppliers have no incentive to offer different from their production costs. Thus, the declared social welfare based on generation offers reflects the true social welfare, and hence, maximizing social welfare is commonly accepted as the right goal in a market setting [2], [3].

However, market-clearing procedures based on declared social welfare maximization are characterized by several practical shortcomings [4], [5]. The assumption of perfect competition does not hold in real-life power markets. Consequently, generation offers may not reflect actual costs and the optimization might not maximize the true social welfare or accurately reflect the natural behavior of market participants. This distortion may be stressed by the presence of nonconvexities related to generation [1], [6] such as nonconvex energy offer curves, no-load offers, ramping rates, minimum generation limits, and minimum up and down times.

This paper analyzes an alternative type of market-clearing procedure for energy trading in an electricity pool. The alternative market clearing explicitly incorporates market-clearing prices in the problem formulation. This procedure is hereinafter referred to as price-based market clearing. Examples of price-based market clearing are 1) procedures based on consumer payment minimization [5], [7]-[14], where market-clearing prices appear in the objective function, and
2) revenue-constrained market-clearing procedures [7]-[10], [12], where market-clearing prices appear in the constraints. The day-ahead auction of the Spanish electricity market [15] is a practical instance of price-based market clearing where generator revenue constraints are considered. Moreover, new procedures explicitly modeling generation and consumer surplus maximization [16] would fall within this class of problems.

The presence of market-clearing prices in the problem formulation complicates the solution of the optimization problem since market-clearing prices may themselves result from an optimization process.

Several definitions for the market-clearing price are available [2], being the two most commonly adopted 1) the price of the highest accepted generation offer, which guarantees revenue adequacy for suppliers, and 2) the marginal price, which provides appropriate economic signals. While the former has received considerable attention in price-based market clearing [5], [7], [9]-[11], [13], few works have considered marginal pricing [8], [12], [14], [16].

In [8], payment minimization under marginal pricing was addressed by a heuristic approach. The proposed auction was based on simple-part supply offers through which generators were assumed to internalize their technical constraints. Therefore, the resulting problem formulation only included a single set of constraints ensuring revenue adequacy to generators.

Hao and Zhuang [12] presented a general formulation for power auctions where marginal prices were used in a consumer payment minimization context. The authors suggested taking advantage of the two-layer structure of the problem formulation but no specific solution algorithm was described.

Zhao et al. [14] were the first to formulate consumer payment minimization under marginal pricing as a bilevel programming problem in which the upper level minimizes consumer payment whereas the lower level determines marginal market-clearing prices. This important result permits the development of mathematical programming solutions to this complex problem. In [14], augmented Lagrangian relaxation and surrogate optimization were proposed, the solution of which might not satisfy feasibility, as stated by the authors, thus requiring heuristic feasibility repair procedures.

In [16], the authors described a new auction driven by consumer and generation surplus maximization based on marginal market-clearing prices. Agent-based simulation was used to gain insight into the performance of the new auction model.

Within the framework of marginal pricing [17], the main purpose of this paper is to formulate a general price-based market-clearing procedure as a bilevel programming problem [18], [19], of which the consumer payment minimization reported in [14] is a particular instance. Bilevel programming is appropriate to model problems where one agent, the leader, optimizes its objective function (upper-level problem) considering that a second agent, the follower, will react by optimizing its own objective function (lower-level problem). These models are relevant in those situations where the actions of the follower affect the decision making of the leader. This is the case in price-based market clearing: the selection of accepted bids and offers (upper-level problem) depends on market-clearing prices (lower-level problem), which are in turn determined based on the set of accepted bids and offers. Under marginal pricing, 
market-clearing prices are the Lagrange multipliers or dual variables associated with the power balance equations in an economic dispatch problem [17].

Unlike previous works [8], [12], [14], [16], the proposed bilevel programming formulation takes into account intertemporal constraints such as ramping rates, minimum up and down times, and time-dependent start-ups. Another salient feature of this paper with respect to [14] is the proposal of an equivalent single-level mixed-integer linear formulation based on the application of the Karush-Kuhn-Tucker (KKT) optimality conditions and the use of well-known linearization schemes. The main advantages of expressing the original bilevel optimization as an equivalent single-level mixed-integer linear program is the guaranteed convergence to the optimal solution in a finite number of steps [20] and the ready availability of efficient commercial branch-and-cut software [21].

The main contributions of this paper are threefold:

1) The ISO is provided with an optimization-based general framework for market clearing explicitly accounting for marginal market-clearing prices in the problem formulation.

2) Bilevel programming is proposed as a suitable solution methodology.

3) The applicability of bilevel programming is illustrated with the solution of an instance of price-based market clearing, namely the consumer payment minimization problem.

It should be noted that the proposed approach may be useful for the ISO to monitor market participants' behavior under alternative market designs and to examine the long-term implications of such trading schemes.

The remainder of this paper is organized as follows. Section II provides a general formulation for price-based market clearing under marginal pricing. Section III presents the solution methodology. Section IV describes the application of the proposed framework to the consumer payment minimization problem. In Section V, numerical results illustrate the performance of the proposed approach. Relevant conclusions are drawn in Section VI. Finally, the complete formulation for the mixed-integer linear equivalent of the consumer payment minimization problem is provided in the Appendix.

\section{PROBLEM Formulation}

The price-based market-clearing procedure can be formulated in a compact way as

$$
\begin{aligned}
& \min _{x, y, \lambda} F(x, y, \lambda) \\
& \text { subject to } \\
& x \in X \\
& G^{e}(x, y)=0 \\
& G^{i}(x, y) \geq 0 \\
& H(x, y, \lambda) \geq 0 \\
& \lambda=\Gamma(x, y)
\end{aligned}
$$

where the vector of scheduling variables $x$ represents the on/off statuses of generating units and the payments for generation start-ups, whereas the vector of dispatching variables $y$ characterizes energy offers and network-related variables.
The objective function (1) may comprise either the declared social welfare, the consumer payment, or the sum of the generation and consumer surplus. Expressions (2), exclusively involving scheduling variables $x$, include integrality constraints, start-up payment constraints, and minimum up and down times. Constraints (3) denote the equalities associated with power balance equations. Constraints (4) model all inequalities involving variables $x$ and $y$ such as power limits and ramping limits. Expressions (5) represent all inequality constraints involving market-clearing prices such as those imposing revenue adequacy. Finally, constraints (6) are the definition of the vector of market-clearing prices.

Under marginal pricing, the vector of market-clearing prices $\lambda$ is defined as the vector of marginal costs [17]. Mathematically, $\lambda$ can be derived from the following economic dispatch problem for the optimal vector of scheduling variables $x^{*}$ :

$$
\begin{aligned}
& \min _{y} f\left(x^{*}, y\right) \\
& \text { subject to } \\
& G^{e}\left(x^{*}, y\right)=0:(\lambda) \\
& G^{i}\left(x^{*}, y\right) \geq 0:(\mu)
\end{aligned}
$$

where the Lagrange multipliers associated with constraints (8) and (9) are in parentheses.

It is worth mentioning that the objective function (7) may be different from that of the price-based market-clearing procedure (1). Moreover, the vector of market-clearing prices $\lambda$ is equal to the vector of Lagrange multipliers or dual variables associated with the power balance equations (8).

As can be noted, the definition of the vector of marketclearing prices under marginal pricing is an optimization problem itself (7)-(9). Thus, the price-based market-clearing procedure (1)-(6) can be recast as the following bilevel programming problem:

$$
\begin{aligned}
& \min _{x} F\left(x, y^{*}, \lambda^{*}\right) \\
& \text { subject to } \\
& x \in X \\
& H\left(x, y^{*}, \lambda^{*}\right) \geq 0
\end{aligned}
$$

where $y^{*}$ and $\lambda^{*}$ are obtained from

$$
\begin{aligned}
& \min _{y} f\left(x^{*}, y\right) \\
& \text { subject to } \\
& G^{e}\left(x^{*}, y\right)=0:(\lambda) \\
& G^{i}\left(x^{*}, y\right) \geq 0:(\mu) .
\end{aligned}
$$

The above bilevel problem consists of an upper-level optimization (10)-(12) and a lower-level optimization (13)-(15). The upper level controls the vector of scheduling variables $x$. Lower-level decision variables comprise the vector of dispatching variables $y$ whereas the vector of market-clearing prices $\lambda$ is an outcome of the lower-level problem.

The goal of the upper level is to minimize the price-based market-clearing objective function (10) evaluated at the optimal values of the lower-level variables, $y^{*}$ and $\lambda^{*}$, subject to expressions exclusively constraining upper-level decision variables $x$ 
(11), to a set of constrained functions with $y$ and $\lambda$ as parameters (12), and to the lower-level optimization (13)-(15).

The lower-level optimization is identical to the economic dispatch problem (7)-(9) associated with the optimal values of the upper-level decision variables $x^{*}$.

In general, problem (10)-(15) is a mixed-integer nonlinear bilevel programming problem. In practice, offers and bids consist of a set of energy blocks with their corresponding prices, thereby yielding piecewise linear functions. Offers and bids may also be submitted as nonlinear curves, typically represented by quadratic functions [22] that can be accurately approximated by a set of tangent lines. This approximation yields piecewise linear functions, which for practical purposes are indistinguishable from the nonlinear models if enough segments are used [22]. Therefore, the lower-level objective function (13) is typically piecewise linear. Analogously, constraints (14) and (15), including power balance equations as well as generation and network limits, are usually modeled as linear expressions. Hence, the lower-level problem (13)-(15) is parameterized in terms of the upper-level decision vector $x$ in such a way that the lowerlevel problem is linear and thus convex. As described next, this feature allows the transformation of problem (10)-(15) into an equivalent single-level mixed-integer program.

\section{SOLUTION APPROACH}

To convert the original bilevel formulation (10)-(15) into an equivalent single-level problem, the lower-level optimization is first replaced by its KKT conditions.

Consider the Lagrangian function associated with the lowerlevel problem (13)-(15) for a given upper-level vector $\hat{x}$ :

$$
L(\hat{x}, y, \lambda, \mu)=f(\hat{x}, y)-\lambda^{T} G^{e}(\hat{x}, y)-\mu^{T} G^{i}(\hat{x}, y) .
$$

The optimal solution to (13)-(15) must satisfy the KKT necessary optimality conditions:

$$
\begin{aligned}
\frac{\partial L(\hat{x}, y, \lambda, \mu)}{\partial y}=\frac{\partial f(\hat{x}, y)}{\partial y}-\left(\frac{\partial G^{e}(\hat{x}, y)}{\partial y}\right)^{T} \lambda- \\
\left(\frac{\partial G^{i}(\hat{x}, y)}{\partial y}\right)^{T} \mu=0 \\
\mu \geq 0 \\
G^{e}(\hat{x}, y)=0 \\
G^{i}(\hat{x}, y) \geq 0 \\
\mu^{T} G^{i}(\hat{x}, y)=0
\end{aligned}
$$

where (17), (18) are the dual feasibility constraints, (19), (20) are the primal feasibility constraints, and (21) expresses the complementary slackness conditions.

Hence, problem (10)-(15) can be reformulated as the following single-level equivalent:

$$
\begin{aligned}
& \min _{x, y, \lambda, \mu} F(x, y, \lambda) \\
& \text { subject to } \\
& x \in X \\
& H(x, y, \lambda) \geq 0
\end{aligned}
$$

$$
\begin{aligned}
& \frac{\partial f(x, y)}{\partial y}-\left(\frac{\partial G^{e}(x, y)}{\partial y}\right)^{T} \lambda-\left(\frac{\partial G^{i}(x, y)}{\partial y}\right)^{T} \mu=0 \\
& \mu \geq 0 \\
& G^{e}(x, y)=0 \\
& G^{i}(x, y) \geq 0 \\
& \mu^{T} G^{i}(x, y)=0 .
\end{aligned}
$$

Problem (22)-(29) falls into the category of what is known in the literature as a mathematical program with equilibrium constraints [19], [23]. As can be seen, the vectors of Lagrange multipliers $\lambda$ and $\mu$ become decision variables of the resulting single-level equivalent. In addition, the following types of nonlinearities may be present in problem (22)-(29):

1) Nonlinear offer and bid curves leading to nonlinear expressions for the generation and consumer surplus in (22). As mentioned above, these nonlinear expressions are replaced by accurate piecewise linear approximations.

2) Bilinear products of market-clearing prices and power consumptions typically involved in (22) when the demand is elastic, and bilinear products of market-clearing prices and power outputs in (24). These sets of nonlinear products can be transformed into equivalent linear expressions based on the KKT conditions as described in [24].

3) Products of scheduling variables $x$, typically binary, and continuous variables $\lambda$ and $\mu$ in (25). These nonlinearities can be equivalently formulated as linear expressions using previously reported integer algebra results [25].

4) Products of Lagrange multipliers and lower-level decision variables $y$ in the complementary slackness conditions (29). As shown by Fortuny-Amat and McCarl [26], complementary slackness conditions can also be formulated as mixed-integer linear programming expressions. It is worth mentioning that under the assumption of linearity of the lower-level problem, complementary slackness conditions (29) can be replaced by the equality associated with the strong duality theorem [27], thereby leading to a more effective linearization in terms of computational performance [28].

Thus, after some algebra, problem (22)-(29) is recast as a mixed-integer linear programming problem suitable for commercially available branch-and-cut software [21].

\section{APPLICATION}

To illustrate the above bilevel programming framework, we now consider an instance of price-based market clearing, namely the payment minimization problem, hereinafter referred to as PM. The model is built on that presented in [13] with the following extensions: 1) generation offers may consist of several energy blocks and include a no-load term and a time-dependent start-up term, which are both accounted for in the payment function, 2) minimum up and down times are considered, and 3) ramping limits comprising ramp-up, ramp-down, start-up, and shut-down ramp rates are modeled. As done in [13], we assume that the demand is inelastic, and transmission network and ancillary services are ignored. Notwithstanding, these simplifications do not alter our main conclusion, and results could be extended to include all of them at the expense 
of an increased notational complexity. For quick reference, PM is formulated as

$$
\begin{aligned}
& \min _{\substack{o_{j t}^{E}, p_{j t}, s_{j t}, v_{j t}, \lambda_{t} \\
\text { subject to }}} \sum_{t \in T}\left[\lambda_{t} D_{t}+\sum_{j \in J}\left(s_{j t}+v_{j t} O_{j t}^{N L}\right)\right] \\
& \sum_{j \in J} p_{j t}=D_{t} ; \quad \forall t \in T \\
& v_{j t}, s_{j t} \in \Omega_{j t} ; \quad \forall j \in J, \forall t \in T \\
& o_{j t}^{E}, p_{j t} \in \Pi_{j t} ; \quad \forall j \in J, \forall t \in T \\
& v_{j t} \in\{0,1\} ; \quad \forall j \in J, \forall t \in T \\
& \lambda_{t}=\frac{\partial\left(\sum_{j \in J} \sum_{t \in T} o_{j t}^{E^{*}}\right)}{\partial D_{t}} ; \quad \forall t \in T
\end{aligned}
$$

The objective function (30) represents the total payment by consumers and comprises three terms, respectively, related to generation energy offers, start-up offers, and no-load offers. Constraints (31) are the power balance equations. Expressions (32) characterize generation constraints exclusively involving scheduling variables, i.e., minimum up and down times as well as start-up payments. Analogously, constraints (33) are related to dispatching variables and model the block structure of energy offers as well as power limits and ramping limits. The interested reader is referred to [3] and [29] for a detailed description of these linear constraints. Constraints (34) impose the integrality of variables $v_{j t}$. Finally, constraints (35) model the marginal pricing setting. For the sake of unit consistency, hourly time periods are considered.

According to Section II, the bilevel programming formulation for problem (30)-(35) is

$$
\min _{s_{j t}, v_{j t}} \sum_{t \in T}\left[\lambda_{t}^{*} D_{t}+\sum_{j \in J}\left(s_{j t}+v_{j t} O_{j t}^{N L}\right)\right]
$$

subject to

$$
\begin{aligned}
& v_{j t}, s_{j t} \in \Omega_{j t} ; \quad \forall j \in J, \forall t \in T \\
& v_{j t} \in\{0,1\} ; \quad \forall j \in J, \forall t \in T
\end{aligned}
$$

where optimal market-clearing prices $\lambda_{t}^{*}$ are obtained from

$$
\begin{aligned}
& \min _{\substack{o_{j t}^{E}, p_{j t} \\
\text { subject to }}} \sum_{j \in J} \sum_{t \in T} o_{j t}^{E} \\
& \sum_{j \in J} p_{j t}=D_{t}:\left(\lambda_{t}\right) ; \quad \forall t \in T \\
& o_{j t}^{E}, p_{j t} \in \Pi_{j t}:\left(\mu_{j t}\right) ; \quad \forall j \in J, \forall t \in T .
\end{aligned}
$$

\begin{tabular}{|c|c|c|c|c|c|}
\hline & \multirow[b]{4}{*}{ Unit } & \multirow{2}{*}{\multicolumn{2}{|c|}{$\mathrm{CM}$}} & \multirow{2}{*}{\multicolumn{2}{|c|}{ PM }} \\
\hline & & & & & \\
\hline & & \multicolumn{2}{|c|}{ Hour } & \multicolumn{2}{|c|}{ Hour } \\
\hline & & 1 & 2 & 1 & 2 \\
\hline \multirow{4}{*}{$p_{j t}(\mathrm{MW})$} & 1 & 50 & 60 & 50 & $\overline{60}$ \\
\hline & 2 & 40 & 60 & 40 & 60 \\
\hline & 3 & 10 & 30 & 0 & 0 \\
\hline & 4 & 0 & 0 & 10 & 30 \\
\hline$\lambda_{t}(\$ / \mathrm{MWh})$ & & 65 & 65 & 30 & 30 \\
\hline Offer cost (\$) & & \multicolumn{2}{|c|}{6050} & \multicolumn{2}{|c|}{6400} \\
\hline Payment (\$) & & \multicolumn{2}{|c|}{16300} & \multicolumn{2}{|c|}{9300} \\
\hline
\end{tabular}

The upper-level optimization (36)-(38) determines the generator on/off statuses $v_{j t}$ and the corresponding start-up payments $s_{j t}$ that minimize the consumer payment. In contrast, the lower-level problem (39)-(41) determines the energy offer costs $o_{j t}^{E}$, the power outputs $p_{j t}$, and the market-clearing prices $\lambda_{t}$ associated with the upper-level scheduling variables $v_{j t}$ by solving a multiperiod economic dispatch based on the minimization of the overall generation energy offer cost. Note that
TABLE I

DATA FOR THE ILLUSTRATIVE EXAMPLE

\begin{tabular}{ccccccccr} 
& \multicolumn{4}{c}{ Hour 1 } & \multicolumn{4}{c}{ Hour 2 } \\
\cline { 2 - 9 } & \multicolumn{3}{c}{$D_{1}=100 \mathrm{MW}$} & \multicolumn{4}{c}{$D_{2}=150 \mathrm{MW}$} \\
\hline \multirow{2}{*}{ Unit } & $\underline{P}_{j 1}$ & $\bar{P}_{j 1}$ & $O_{1 j 1}^{E}$ & $O_{j 1}^{S U}$ & $\underline{P}_{j 2}$ & $\bar{P}_{j 2}$ & $O_{1 j 2}^{E}$ & $O_{j 2}^{S U}$ \\
& $(\mathrm{MW})$ & $(\mathrm{MW})$ & $(\$ / \mathrm{MWh})$ & $(\$)$ & $(\mathrm{MW})$ & $(\mathrm{MW})$ & $(\$ / \mathrm{MWh})$ & $(\$)$ \\
\hline 1 & 5 & 50 & 10 & 0 & 5 & 60 & 15 & 0 \\
2 & 5 & 40 & 20 & 0 & 5 & 60 & 20 & 0 \\
3 & 0 & 10 & 65 & 50 & 0 & 30 & 65 & 50 \\
4 & 5 & 60 & 30 & 1800 & 5 & 100 & 30 & 1800 \\
\hline
\end{tabular}

TABLE II

RESULTS FOR THE ILLUSTRATIVE EXAMPLE

the lower-level problem (39)-(41) is a linear program parameterized in terms of $v_{j t}$. This parameterization is embedded in the feasibility set $\Pi_{j t}$.

As described in Section III, the use of the KKT optimality conditions, duality theory, and integer algebra results allows transforming the bilevel programming problem (36)-(41) into an equivalent single-level mixed-integer linear program. The complete formulation of the resulting single-level equivalent is provided in the Appendix.

\section{NUMERICAL RESULTS}

Results from several case studies are presented in this section. For didactical purposes, the bilevel programming framework for PM has been first applied to an illustrative example presented in [13]. The second case study is based on the IEEE Reliability Test System (RTS) [30], [31], and includes intertemporal constraints and multiple-block generation offers. In order to assess the practical applicability of the proposed framework, the 25-unit system described in [13] has been replicated to analyze systems including up to 100 generating units. For all cases, the results of PM are compared with those achieved by a conventional market-clearing procedure based on the minimization of the generation offer cost including energy, start-up, and no-load offers [3]. This cost minimization problem is denoted as CM. The model has been implemented on a Dell PowerEdge R910 X64 with four processors at $8 \mathrm{GHz}$ and $32 \mathrm{~GB}$ of RAM using CPLEX 12.1 [21] under GAMS [32].

\section{A. Illustrative Example}

The first test case was described in [13] and considers $2 \mathrm{~h}$ and four generating units which are initially scheduled off. Generation offers comprise a single energy block and a single start-up price, whereas no-load offers, minimum up and down times as well as ramping limits are not modeled. Table I shows the data 
TABLE III

GeNERATION DATA FOR THE RTS-BASED CASE

\begin{tabular}{lcrcccccccrrr}
\hline $\begin{array}{c}\text { Unit } \\
\text { group }\end{array}$ & $\begin{array}{c}\text { Number } \\
\text { of units }\end{array}$ & $\begin{array}{c}\underline{P}_{j t} \\
(\mathrm{MW})\end{array}$ & $\begin{array}{c}\bar{P}_{j t} \\
(\mathrm{MW})\end{array}$ & $\begin{array}{c}R U_{j} \\
(\mathrm{MW} / \mathrm{h})\end{array}$ & $\begin{array}{c}R D_{j} \\
(\mathrm{MW} / \mathrm{h})\end{array}$ & $\begin{array}{c}S U_{j} \\
(\mathrm{MW} / \mathrm{h})\end{array}$ & $\begin{array}{c}S D_{j} \\
(\mathrm{MW} / \mathrm{h})\end{array}$ & $\begin{array}{c}U T_{j} \\
(\mathrm{~h})\end{array}$ & $\begin{array}{c}D T_{j} \\
(\mathrm{~h})\end{array}$ & $\begin{array}{c}S_{j}^{0} \\
(\mathrm{~h})\end{array}$ & $\begin{array}{c}O_{j t}^{S U} \\
(\$)\end{array}$ & $\begin{array}{c}O_{j t}^{N L} \\
(\$ / \mathrm{h})\end{array}$ \\
\hline U12 & 5 & 2.40 & 12 & 12 & 12 & 12 & 12 & 4 & 2 & 2 & 87.4 & 56.19 \\
$\mathrm{U} 20$ & 4 & 15.80 & 20 & 20 & 20 & 20 & 20 & 1 & 1 & 1 & 15.0 & 467.32 \\
U50 & 6 & 0.00 & 50 & 50 & 50 & 50 & 50 & 1 & 1 & 1 & 0.0 & 0.00 \\
U76 & 4 & 15.20 & 76 & 76 & 76 & 76 & 76 & 8 & 4 & 4 & 715.2 & 174.16 \\
U100 & 3 & 25.00 & 100 & 100 & 100 & 100 & 100 & 8 & 8 & 8 & 575.0 & 465.12 \\
U155 & 4 & 54.25 & 155 & 155 & 155 & 155 & 155 & 8 & 8 & 8 & 312.0 & 538.05 \\
U197 & 3 & 68.95 & 197 & 180 & 180 & 180 & 180 & 12 & 10 & 10 & 1018.9 & 1323.87 \\
U350 & 1 & 140.00 & 350 & 240 & 240 & 240 & 240 & 24 & 48 & 24 & 2298.0 & 1411.54 \\
U400 & 2 & 100.00 & 400 & 400 & 400 & 400 & 400 & 1 & 1 & 1 & 0.0 & 530.88 \\
\hline
\end{tabular}

for both periods. This example is useful to highlight the differences in the results yielded by PM and CM.

For this illustrative example, the computing time required to achieve the optimal solution to PM using the single-level equivalent was less than $1 \mathrm{~s}$.

The optimal solutions to CM and PM are summarized in Table II. Under conventional market clearing, generators 1, 2, and 3 are dispatched at maximum capacity in both periods, whereas generator 4 is not scheduled. Generator 3 is the marginal unit in both periods thereby setting the corresponding market-clearing prices equal to its energy offer price, i.e., $\$ 65 / \mathrm{MWh}$. This solution costs $\$ 6050$ and yields a payment equal to $\$ 16300$.

In contrast, PM results in a different schedule in both periods. While generators 1 and 2 do not experience any change in their generation levels with respect to the solution to CM, generators 3 and 4 exchange their schedules and power dispatches. As a consequence, generator 4 becomes the marginal unit in both periods, setting both market-clearing prices at $\$ 30 / \mathrm{MWh}$. The optimal payment is equal to $\$ 9300$ and the associated offer cost is equal to $\$ 6400$. In other words, a $42.9 \%$ reduction in payment is attained at the expense of a 5.8\% increase in offer cost.

In this case, the market-clearing price in each period under marginal pricing is identical to the corresponding highest accepted energy offer price. Note, however, that both pricing schemes lead in general to different market-clearing prices irrespective of the objective function being minimized. As an example, let the demand at hour 1 be reduced so that it belongs to the interval $(50,55] \mathrm{MW}$. At the optimal solution to either CM or PM, generator 2 would be dispatched at its minimum power output while generator 1 would be the marginal unit by supplying the remaining demand. Under marginal pricing, the market-clearing price would be the energy offer price of generator 1, i.e., $\$ 10 / \mathrm{MWh}$, whereas the highest accepted energy offer price is that of generator 2, i.e., $\$ 20 / \mathrm{MWh}$.

\section{B. RTS-Based Case}

The second case study considers 32 generating units and $24 \mathrm{~h}$. Table III lists the data for generators including power limits, ramping rates, minimum up and down times, initial statuses, and start-up and no-load offer prices. It is assumed that generating units submit piecewise linear energy offers consisting of as many slopes as the number of incremental heat rate blocks (see [31, Table IX]). The conversion rates from Btu to dollars for each type of fuel are from [30], i.e., \#6 oil \$2.3/MBtu,
TABLE IV

SYSTEM DEMAND FOR THE RTS-BASED CASE

\begin{tabular}{rccc}
\hline$t$ & $D_{t}(\mathrm{MW})$ & $t$ & $D_{t}(\mathrm{MW})$ \\
\hline 1 & 1277.46 & 13 & 1885.78 \\
2 & 1257.19 & 14 & 1865.50 \\
3 & 1216.63 & 15 & 1824.95 \\
4 & 1176.08 & 16 & 1784.39 \\
5 & 1196.35 & 17 & 1824.95 \\
6 & 1318.02 & 18 & 1865.50 \\
7 & 1459.96 & 19 & 1946.61 \\
8 & 1723.56 & 20 & 1987.16 \\
9 & 1926.33 & 21 & 1946.61 \\
10 & 2007.44 & 22 & 1824.95 \\
11 & 2027.72 & 23 & 1622.17 \\
12 & 2007.44 & 24 & 1419.40 \\
\hline
\end{tabular}

TABLE V

RESULTS FOR THE RTS-BASED CASE

\begin{tabular}{ccc}
\hline & PM & CM \\
\hline Payment (\$) & 537681 & 650832 \\
Offer cost (\$) & 384852 & 336867 \\
\hline
\end{tabular}

\#2 oil \$3.0/MBtu, coal \$1.2/MBtu, and nuclear \$0.6/MBtu, respectively. Moreover, it is also assumed that generators do not modify their respective supply offers over the time span. The hourly system demand is shown in Table IV and corresponds to Wednesday of week 35 [31].

In a practical operational setting, proving optimality is less important than improving the current solution within a specific timeframe. Thus, based on current industry practice [33], the execution of CPLEX was stopped when the value of the payment was below a specified threshold or when this number appeared to have reached a lower bound. In addition, a time limit of $1 \mathrm{~h}$ was set. With these stopping criteria, Table V shows the results attained by the proposed approach for PM. The computing time required by this solution was $44.5 \mathrm{~min}$. Table $\mathrm{V}$ also lists the results corresponding to the optimal solution to $\mathrm{CM}$, which was attained in $13.3 \mathrm{~s}$. Note that the payment associated with the optimal solution to CM represents a relevant upper bound for the optimal objective function value of PM. As can be seen, a substantial $17.4 \%$ reduction in payment is achieved by increasing the offer cost by $14.2 \%$.

Moreover, the stopping criteria have been validated by observing the evolution of the objective function value versus computing time when longer executions are implemented. Model runs for PM within time limits of $3600 \mathrm{~s}, 10000 \mathrm{~s}$, and $50000 \mathrm{~s}$ were carried out, and total payments obtained were found to be within $0.01 \%$ and $0.003 \%$ of each other, respectively, whereas 


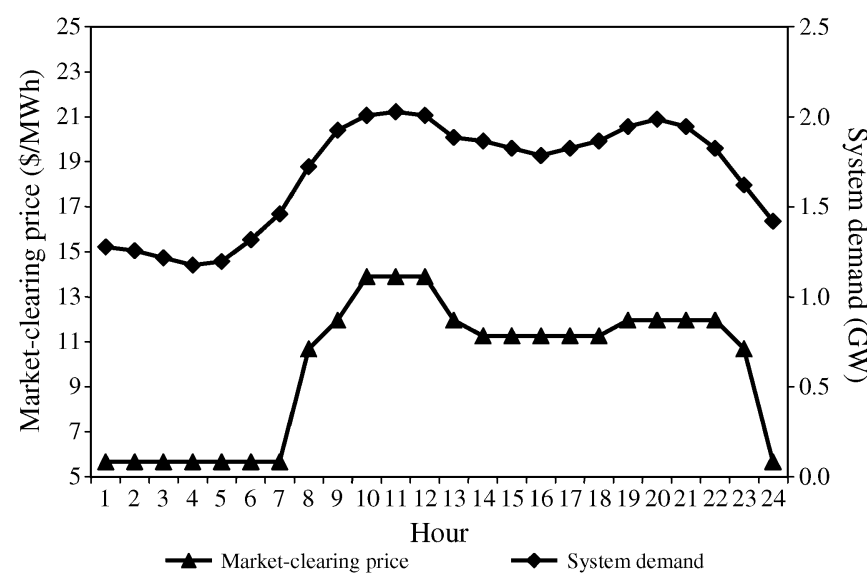

Fig. 1. Market-clearing prices and system demand for the RTS-based case.

the system operation was similar for all simulations. Hence, the stopping criteria adopted based on a 1-h timeframe were judged to provide an adequate tradeoff between computing time and solution accuracy.

Hourly market-clearing prices associated with the solution to PM presented in Table V are depicted in Fig. 1. This figure also shows the hourly system demand. Note that market-clearing prices determined by PM follow the shape of the demand curve. This is a reasonable result bearing in mind that market-clearing prices are the Lagrange multipliers associated with the power balance equations in the economic dispatch characterizing the lower-level problem.

\section{Real-Sized Case Studies}

The proposed bilevel programming formulation has been applied to solve several real-sized case studies built on a base test system comprising 25 generating units. A time span of $24 \mathrm{~h}$ is considered. The data for the generating units and demand of the base test system can be found in [13]. Three additional case studies have been generated by replicating the base test system and scaling the system demand accordingly.

Table VI lists the results obtained by PM with the aforementioned stopping criteria. The results associated with the optimal solutions to CM are also provided in Table VI. As can be seen, payments are reduced by factors ranging between $6.99 \%$ for the 25 -unit case and $7.28 \%$ for the 100-unit case with respect to the corresponding upper bound provided by the optimal solution to CM. These payment reductions incur slight cost increases below $2.6 \%$ for all cases. It is worth mentioning that the computing times required to attain such near-optimal solutions are significantly lower than that required for the RTS-based case. This is an indication of the case-dependent behavior of the branch-and-cut algorithm. The quality of the solutions attained by PM has been assessed by allowing longer execution times up to $50000 \mathrm{~s}$. In all extended simulations, total payments found by PM remained unchanged with respect to the values reported in Table VI, thereby supporting the validity of the results.

\section{CONCLUSION}

This paper has formulated the price-based market-clearing problem under marginal pricing as a general bilevel programming problem, offering flexibility in the problem definition. The resulting bilevel programming formulation is transformed into
TABLE VI

RESULTS FOR THE REAL-SIZED TEST SySTEMS

\begin{tabular}{cccc}
\hline \# of units & & PM & CM \\
\hline \multirow{3}{*}{25} & Payment (\$) & 4764845 & 5122905 \\
& Offer cost (\$) & 3482645 & 3394415 \\
& Computing time (s) & 3.23 & 0.27 \\
\hline \multirow{3}{*}{50} & Payment (\$) & 9529690 & 10262810 \\
& Offer cost (\$) & 6964970 & 6788750 \\
& Computing time (s) & 11.87 & 0.22 \\
\hline \multirow{4}{*}{75} & Payment (\$) & 14294535 & 15394165 \\
& Offer cost (\$) & 10447935 & 10183055 \\
& Computing time (s) & 24.47 & 0.54 \\
\hline \multirow{3}{*}{100} & Payment (\$) & 19059380 & 20556770 \\
& Offer cost (\$) & 13930580 & 13577410 \\
& Computing time (s) & 52.56 & 0.41 \\
\hline \multirow{2}{*}{} & & &
\end{tabular}

an equivalent single-level mixed-integer linear program. This transformation comprises two steps. First, the lower-level optimization is replaced by a set of constraints based on the KKT optimality conditions. Subsequently, a number of nonlinearities are converted to linear equivalents using some well-known linearization schemes.

The ultimate goal of this paper is to provide the ISO with a tool that can be used for comparative analysis of different market-clearing procedures, so that informed decisions can be made.

This general bilevel formulation and its single-level equivalent have been applied to an instance of price-based market clearing in which consumer payment is minimized. Numerical results reveal that bilevel programming is an effective approach to address price-based market-clearing procedures.

We are currently investigating new formulations of the singlelevel equivalent model with the goal of reducing the computation time required to prove optimality. Moreover, research is underway to solve other instances of price-based market clearing such as those explicitly maximizing the surplus of consumers and producers, as well as market-clearing procedures with revenue adequacy constraints. Another interesting avenue of research is to analyze the long-term effects of price-based market clearing and to monitor the behavior of market agents under alternative market designs. Finally, further research will also be devoted to the analysis of joint energy and reserve electricity markets.

\section{APPENDIX}

The single-level equivalent of the consumer payment minimization problem presented in Section IV can be formulated as the following mixed-integer linear program:

$$
\min _{\substack{a_{j t}, \tilde{a}_{j t}, b_{j t}, \tilde{b}_{j t}, c_{j t}, \tilde{c}_{j t}, d_{j t}, \tilde{d}_{j t}, e_{j t}, \tilde{e}_{j t}, k_{j t}, \tilde{k}_{j t}, n_{j t}, \tilde{n}_{j t}, o_{j t}, p_{j t}, r_{j t}, \tilde{r}_{j t}, s_{j t}, v_{j t}, \beta_{b j t}, \gamma_{j t}, \epsilon_{j t}, \theta_{j t}, \lambda_{t}, \xi_{j t}, \sigma_{j t}}}\left[\lambda_{t} D_{t}+\sum_{j \in J}\left(s_{j t}+v_{j t} O_{j t}^{N L}\right)\right]
$$

subject to

$v_{j t}, s_{j t} \in \Omega_{j t} ; \forall j \in J, \forall t \in T$ 


$$
\begin{aligned}
& v_{j t} \in\{0,1\} ; \forall j \in J, \forall t \in T \\
& \sum_{j \in J} p_{j t}=D_{t} ; \forall t \in T \\
& o_{j t}^{E}, p_{j t} \in \Pi_{j t} ; \forall j \in J, \forall t \in T \\
& \lambda_{t}+\gamma_{j t}+\theta_{j t}+\xi_{j t}-\xi_{j t+1} \\
& +\sigma_{j t}-\sigma_{j t+1}+\epsilon_{j t}=0 ; \forall j \in J, \forall t=1 \ldots n_{T}-1 \\
& \lambda_{n_{T}}+\gamma_{j n_{T}}+\theta_{j n_{T}}+\xi_{j n_{T}}+\sigma_{j n_{T}}=0 ; \forall j \in J \\
& -\gamma_{j t}+\beta_{b j t} \leq O_{b j t}^{E} ; \forall b=2 \ldots n_{B_{j}}, \forall j \in J, \forall t \in T \\
& \theta_{j t} \leq 0 ; \forall j \in J, \forall t \in T \\
& \xi_{j t} \leq 0 ; \forall j \in J, \forall t \in T \\
& \sigma_{j t} \geq 0 ; \forall j \in J, \forall t \in T \\
& \epsilon_{j t} \leq 0 ; \forall j \in J, \forall t=1 \ldots n_{T}-1 \\
& \beta_{b j t} \leq 0 ; \forall b=2 \ldots n_{B_{j}}, \forall j \in J, \forall t \in T \\
& \sum_{t \in T} \lambda_{t} D_{t}+\sum_{j \in J} \sum_{t \in T}\left(a_{j t} \bar{P}_{j t}+b_{j t} \underline{P}_{j t}\right) \\
& +\sum_{j \in J}\left[\xi_{j 1} P_{j 0}+\xi_{j 1} R U_{j} V_{j 0}+\left(c_{j 1}-\xi_{j 1} V_{j 0}\right) S U_{j}\right. \\
& \left.+\left(\xi_{j 1}-c_{j 1}\right) \bar{P}_{j 1}\right] \\
& +\sum_{j \in J} \sum_{t=2}^{n_{T}}\left[d_{j t} R U_{j}+\left(c_{j t}-d_{j t}\right) S U_{j}+\left(\xi_{j t}-c_{j t}\right) \bar{P}_{j t}\right] \\
& +\sum_{j \in J}\left[\sigma_{j 1} P_{j 0}-n_{j 1} R D_{j}-\left(\sigma_{j 1} V_{j 0}-n_{j 1}\right) S D_{j}\right. \\
& \left.-\sigma_{j 1} \bar{P}_{j 1}\left(1-V_{j 0}\right)\right] \\
& -\sum_{j \in J} \sum_{t=2}^{n_{T}}\left[n_{j t} R D_{j}+\left(r_{j t}-n_{j t}\right) S D_{j}+\left(\sigma_{j t}-r_{j t}\right) \bar{P}_{j t}\right] \\
& +\sum_{j \in J} \sum_{t=1}^{n_{T}-1}\left[\left(e_{j t}-k_{j t}\right) S D_{j}+k_{j t} \bar{P}_{j t}\right] \\
& +\sum_{j \in J} \sum_{t \in T} v_{j t} \underline{P}_{j t} O_{1 j t}^{E}+\sum_{b=2}^{n_{B_{j}}} \sum_{j \in J} \sum_{t \in T} \beta_{b j t} \bar{P}_{b j t} \\
& =\sum_{j \in J} \sum_{t \in T} o_{j t}^{E} \\
& \tilde{a}_{j t}=\theta_{j t}-a_{j t} ; \forall j \in J, \forall t \in T \\
& v_{j t} \underline{\theta}_{j t} \leq a_{j t} \leq 0 ; \forall j \in J, \forall t \in T \\
& \left(1-v_{j t}\right) \underline{\theta}_{j t} \leq \tilde{a}_{j t} \leq 0 ; \forall j \in J, \forall t \in T \\
& \tilde{b}_{j t}=\gamma_{j t}-b_{j t} ; \forall j \in J, \forall t \in T \\
& v_{j t} \underline{\gamma}_{j t} \leq b_{j t} \leq v_{j t} \bar{\gamma}_{j t} ; \forall j \in J, \forall t \in T \\
& \left(1-v_{j t}\right) \underline{\gamma}_{j t} \leq \tilde{b}_{j t} \leq\left(1-v_{j t}\right) \bar{\gamma}_{j t} ; \forall j \in J, \forall t \in T \\
& \tilde{c}_{j t}=\xi_{j t}-c_{j t} ; \forall j \in J, \forall t \in T \\
& v_{j t} \underline{\xi}_{j t} \leq c_{j t} \leq 0 ; \forall j \in J, \forall t \in T \\
& \left(1-v_{j t}\right) \underline{\xi}_{j t} \leq \tilde{c}_{j t} \leq 0 ; \forall j \in J, \forall t \in T \\
& \tilde{d}_{j t}=\xi_{j t}-d_{j t} ; \forall j \in J, \forall t=2 \ldots n_{T} \\
& v_{j t-1} \underline{\xi}_{j t} \leq d_{j t} \leq 0 ; \forall j \in J, \forall t=2 \ldots n_{T} \\
& \left(1-v_{j t-1}\right) \underline{\xi}_{j t} \leq \tilde{d}_{j t} \leq 0 ; \forall j \in J, \forall t=2 \ldots n_{T} \\
& \tilde{n}_{j t}=\sigma_{j t}-n_{j t} ; \forall j \in J, \forall t \in T \\
& 0 \leq n_{j t} \leq v_{j t} \bar{\sigma}_{j t} ; \forall j \in J, \forall t \in T
\end{aligned}
$$

$$
\text { The above formulation is based on those presented in [3] and }
$$
[29]. Parameter $\bar{P}_{b j t}$ represents the upper bound for the power produced from the $b$ th energy block offered by unit $j$ in period $t$. The number of energy blocks offered by unit $j$ is denoted by $n_{B_{j}}$. Similarly, $n_{T}$ is the number of time periods. It should also be noted that the vector of Lagrange multipliers $\mu_{j t}$ associated with constraints (41) is made up of $\beta_{b j t}$, associated with the upper bound for the power produced in each energy block; $\gamma_{j t}$, associated with the definition of $p_{j t}$ as the sum of the power produced in each energy block; $\epsilon_{j t}$, related to the shut-down ramping rate constraints; $\theta_{j t}$, associated with the upper bound for the power output; $\xi_{j t}$, associated with the ramp-up and start-up ramp rate constraints; and $\sigma_{j t}$, related to the ramp-down constraints. Symbols $\bar{\gamma}_{j t}$ and $\bar{\sigma}_{j t}$ are the respective upper bounds for $\gamma_{j t}$ and $\sigma_{j t}$. Analogously, $\underline{\gamma}_{j t}, \underline{\epsilon}_{j t}, \underline{\theta}_{j t}$, and $\underline{\xi}_{j t}$ are the lower bounds for $\gamma_{j t}, \epsilon_{j t}, \theta_{j t}$, and $\xi_{j t}$, respectively. As described in [24], these bounding parameters may be selected based on the values of the corresponding Lagrange multipliers resulting from the optimal solution to the associated offer cost minimization problem.

The objective function (42) and constraints (43), (44) correspond to the upper-level optimization (36)-(38) whereas (45)-(79) equivalently replace the lower-level optimization problem (39)-(41). Constraints (45), (46) are the primal feasibility constraints, (47)-(54) represent the dual feasibility constraints, (55) expresses the linear equality associated with the strong duality theorem, which represents complementary slackness conditions, and (56)-(79) model the linearization of the products of binary and continuous variables resulting from the transformation to a single-level equivalent. These linear terms require the introduction of additional continuous variables $a_{j t}, \tilde{a}_{j t}, b_{j t}, \tilde{b}_{j t}, c_{j t}, \tilde{c}_{j t}, d_{j t}, \tilde{d}_{j t}, e_{j t}, \tilde{e}_{j t}, k_{j t}, \tilde{k}_{j t}$, $n_{j t}, \tilde{n}_{j t}, r_{j t}$, and $\tilde{r}_{j t}$.

\section{REFERENCES}

[1] A. J. Wood and B. F. Wollenberg, Power Generation, Operation, and Control, 2nd ed. New York: Wiley, 1996.

[2] M. Shahidehpour, H. Yamin, and Z. Li, Market Operations in Electric Power Systems. New York: Wiley, 2002.

[3] J. M. Arroyo and A. J. Conejo, "Multiperiod auction for a pool-based electricity market," IEEE Trans. Power Syst., vol. 17, no. 4, pp. 1225-1231, Nov. 2002.

[4] J. M. Jacobs, "Artificial power markets and unintended consequences," IEEE Trans. Power Syst., vol. 12, no. 2, pp. 968-972, May 1997.

[5] G. A. Stern, J. H. Yan, P. B. Luh, and W. E. Blankson, "What objective function should be used for optimal auctions in the ISO/RTO electricity market?," presented at the IEEE PES General Meeting, Montreal, QC, Canada, Jun. 2006. 
[6] F. D. Galiana, A. L. Motto, and F. Bouffard, "Reconciling social welfare, agent profits, and consumer payments in electricity pools," IEEE Trans. Power Syst., vol. 18, no. 2, pp. 452-459, May 2003.

[7] S. Hao, G. A. Angelidis, H. Singh, and A. D. Papalexopoulos, "Consumer payment minimization in power pool auctions," IEEE Trans. Power Syst., vol. 13, no. 3, pp. 986-991, Aug. 1998.

[8] J. Alonso, A. Trías, V. Gaitan, and J. J. Alba, "Thermal plant bids and market clearing in an electricity pool. Minimization of costs vs. minimization of consumer payments," IEEE Trans. Power Syst., vol. 14, no. 4, pp. 1327-1334, Nov. 1999.

[9] C. Vázquez, M. Rivier, and I. J. Pérez-Arriaga, "Production cost minimization versus consumer payment minimization in electricity pools," IEEE Trans. Power Syst., vol. 17, no. 1, pp. 119-127, Feb. 2002.

[10] Y. Ren and F. D. Galiana, "Minimum consumer payment scheduling and pricing in electricity markets," presented at the 14th Power Systems Computation Conf., PSCC'02, Sevilla, Spain, Jun. 2002.

[11] D. P. Mendes, "Resource scheduling and pricing in a centralised energy market," presented at the 14th Power Systems Computation Conf., PSCC'02, Sevilla, Spain, Jun. 2002.

[12] S. Hao and F. Zhuang, "New models for integrated short-term forward electricity markets," IEEE Trans. Power Syst., vol. 18, no. 2, pp. 478-485, May 2003.

[13] P. B. Luh, W. E. Blankson, Y. Chen, J. H. Yan, G. A. Stern, S.-C. Chang, and F. Zhao, "Payment cost minimization auction for deregulated electricity markets using surrogate optimization," IEEE Trans. Power Syst., vol. 21, no. 2, pp. 568-578, May 2006.

[14] F. Zhao, P. B. Luh, J. H. Yan, G. A. Stern, and S.-C. Chang, "Payment cost minimization auction for deregulated electricity markets with transmission capacity constraints," IEEE Trans. Power Syst., vol. 23, no. 2, pp. 532-544, May 2008.

[15] The OMEL Website. 2011 [Online]. Available: http://www.omel.es/.

[16] A. Somani and L. Tesfatsion, "An agent-based test bed study on wholesale power market performance measures," IEEE Comput. Intell. Mag., vol. 3, no. 4, pp. 56-72, Nov. 2008.

[17] F. C. Schweppe, M. C. Caramanis, R. D. Tabors, and R. E. Bohn, Spot Pricing of Electricity. Norwell, MA: Kluwer, 1988

[18] J. F. Bard, Practical Bilevel Optimization. Algorithms and Applications. Dordrecht, The Netherlands: Kluwer, 1998.

[19] S. Dempe, Foundations of Bilevel Programming. Dordrecht, The Netherlands: Kluwer, 2002.

[20] G. L. Nemhauser and L. A. Wolsey, Integer and Combinatorial Optimization. New York: Wiley-Interscience, 1999.

[21] The IBM ILOG CPLEX Website, 2011. [Online]. Available: http:// www-01.ibm.com/software/integration/optimization/cplex-optimizer.

[22] J. Wang, N. E. Redondo, and F. D. Galiana, "Demand-side reserve offers in joint energy/reserve electricity markets," IEEE Trans. Power Syst., vol. 18, no. 4, pp. 1300-1306, Nov. 2003.

[23] Z.-Q. Luo, J.-S. Pang, and D. Ralph, Mathematical Programs with Equilibrium Constraints. Cambridge, U.K.: Cambridge Univ. Press, 1996.

[24] C. Ruiz and A. J. Conejo, "Pool strategy of a producer with endogenous formation of locational marginal prices," IEEE Trans. Power Syst., vol. 24, no. 4, pp. 1855-1866, Nov. 2009.

[25] C. A. Floudas, Nonlinear and Mixed-Integer Optimization: Fundamentals and Applications. New York: Oxford Univ. Press, 1995.

[26] J. Fortuny-Amat and B. McCarl, "A representation and economic interpretation of a two-level programming problem," J. Oper. Res. Soc., vol. 32, no. 9, pp. 783-792, Sep. 1981.

[27] D. G. Luenberger, Linear and Nonlinear Programming, 2nd ed. New York: Wiley, 1989.
[28] J. M. Arroyo, "Bilevel programming applied to power system vulnerability analysis under multiple contingencies," IET Gen., Transm., Distrib., vol. 4, no. 2, pp. 178-190, Feb. 2010.

[29] M. Carrión and J. M. Arroyo, "A computationally efficient mixed-integer linear formulation for the thermal unit commitment problem," IEEE Trans. Power Syst., vol. 21, no. 3, pp. 1371-1378, Aug. 2006.

[30] R. Billinton and W. Li, Reliability Assessment of Electric Power Systems Using Monte Carlo Methods. New York: Plenum, 1994.

[31] Reliability Test System Task Force, "The IEEE Reliability Test System-1996," IEEE Trans. Power Syst., vol. 14, no. 3, pp. 1010-1020, Aug. 1999.

[32] The GAMS Development Corporation Website, 2011. [Online]. Available: http://www.gams.com.

[33] R. Sioshansi, R. O’Neill, and S. S. Oren, "Economic consequences of alternative solution methods for centralized unit commitment in dayahead electricity markets," IEEE Trans. Power Syst., vol. 23, no. 2, pp. 344-352, May 2008.

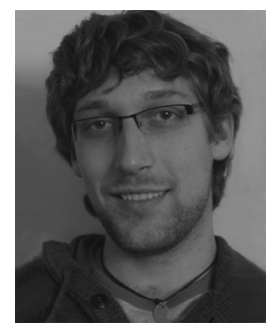

Ricardo Fernández-Blanco (S'10) received the Ingeniero Industrial degree from the Universidad de Castilla-La Mancha, Ciudad Real, Spain, in 2009. $\mathrm{He}$ is currently pursuing the Ph.D. degree at the Universidad de Castilla-La Mancha.

His research interests are in the fields of power systems economics, bilevel programming, and electricity markets.



José M. Arroyo (S'96-M'01-SM'06) received the Ingeniero Industrial degree from the Universidad de Málaga, Málaga, Spain, in 1995, and the Ph.D. degree in power systems operations planning from the Universidad de Castilla-La Mancha, Ciudad Real, Spain, in 2000

From June 2003 through July 2004, he held a Richard H. Tomlinson Postdoctoral Fellowship at the Department of Electrical and Computer Engineering of McGill University, Montreal, QC, Canada. He is currently a Full Professor of electrical engineering at the Universidad de Castilla-La Mancha. His research interests include operations, planning, and economics of power systems, as well as optimization and parallel computation.

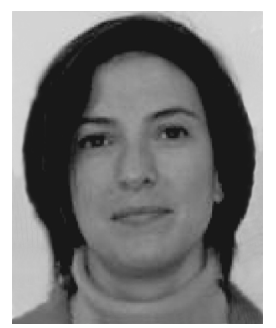

Natalia Alguacil (S'97-M'01-SM'07) received the Ingeniero en Informática degree from the Universidad de Málaga, Málaga, Spain, in 1995, and the Ph.D. degree in power systems operations and planning from the Universidad de Castilla-La Mancha, Ciudad Real, Spain, in 2001.

She is currently an Associate Professor of electrical engineering at the Universidad de Castilla-La Mancha. Her research interests include operations, planning, and economics of power systems, as well as optimization. 